\title{
Le cancer du sein et l'irradiation : Le point des connaissances
}

\author{
Mme S. VIGNES * \\ (Manuscrit reçu le 26 janvier 1982) \\ RÉSUMÉ
}

Le cancer du sein, cancer le plus fréquent chez la femme, peut être provoqué par de nombreux agents physiques ou chimiques.

La contribution des rayonnements ionisants a été clairement démontrée à la fois par des experimentations animales sur les rats et des enquêtes épidémiologiques sur des groupes de femmes exposées à des irradiations médicales, ou survivantes des explosions japonaises. Le risque moyen par unité de dose (rad) est estimé, de l'ordre de 6 par an et par million de femmes. Ce risque peut varier beaucoup selon l'âge de la femme à l'exposition et d'autres facteurs soit internes (principalement hormonaux) ou externes (alimentation par exemple).

En radioprotection, le sein constitue un tissu à haut risque, ce qui explique le facteur élevé de risque retenu par la CIPR pour ce tissu.

\section{ABSTRACT}

Breast cancer, the most frequent in females, can be induced by many physical and chemical agents. The contribution of ionizing radiation has been clearly demonstrated both by animal experiments on rats and epidemiologic surveys on populations of women submitted to medical exposure or on Japanese atomic bomb survivors. The mean risk per dose unit (rad) is estimated at about 6 per year and per $10^{6}$ females. It can vary widely with the age at the time of exposure and other internal (mainly hormonal) or external (diet for instance) factors. For radiation protection purposes, breast is a high risk tissue, which explains the high risk factor adopted by ICRP for this tissue.

\section{INTRODUCTION}

Le sein apparaît comme un tissu très cancérisable. Le cancer du sein constitue la première cause de mortalité par cancer chez la femme en France, représentant 16 à $17 \%$ des décès par cancers. Ce taux reste bien inférieur à l'incidence réelle des cancers mammaires, puisque près de $50 \%$ des cancers du sein sont guérissables. Sa fréquence est encore plus élevée dans certains pays comme les Etats-Unis, alors qu'elle est

\footnotetext{
* Electricité de France, Comité de radioprotection, 39, rue de Washington, 75008 Paris.
} 
nettement plus basse dans d'autres, comme le Japon (fig. 1). Le mode de vie et notamment l'alimentation ont souvent été invoqués pour expliquer ces différences. En effet, si des prédispositions héréditaires étaient seules déterminantes, on ne pourrait comprendre pourquoi des femmes japonaises nées aux Etats-Unis et ayant adopté le mode de vie américain voient leur risque de faire un cancer du sein devenir aussi élevé que celui des Américaines.

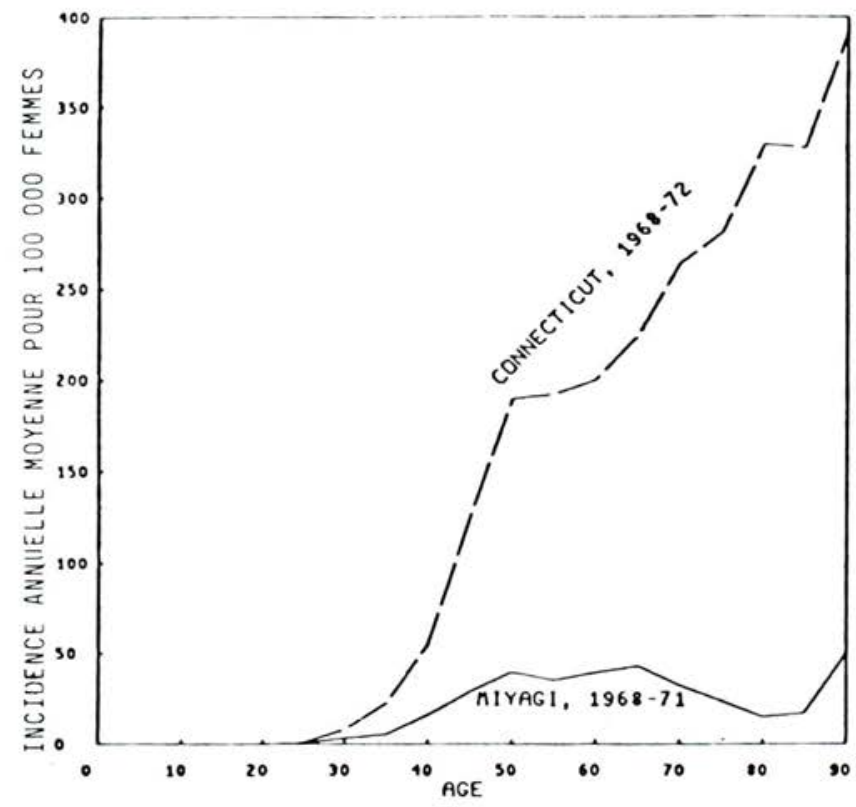

Fig. 1. - Comparaison des taux de cancers du sein aux Etats-Unis et au Japon (selon Land et Coll. [10])

La glande mammaire s'avère finalement être la cible idéale pour tous les types d'agents cancérogènes connus, qu'ils soient endogènes ou exogènes, de nature physique, chimique, virale ou génétique. Rappelons que, dès 1936, Bittner a montré le rôle joué par les virus dans la cancérogénèse mammaire de la souris. Parmi les agents physiques, les rayonnements ionisants se sont révélés des agents cancérogènes possibles, à tel point qu'actuellement, en radioprotection, le sein est considéré comme un tissu "à haut risque", plus encore que la moelle osseuse et la thyroïde. Nous avons aujourd'hui réuni une somme suffisamment importante de données, en provenance soit de l'expérimentation animale soit de l'étude épidémiologique humaine pour permettre de mieux appréhender la contribution des rayonnements à la cancérogénèse mammaire.

Le but de ce court article est d'essayer de faire le point sur cette question. 


\section{RADIOSENSIBILITÉ DE LA GLANDE MAMMAIRE ET SA SUSCEPTIBILITÉ À LA RADIOCANCÉROGÉNĖSE}

\subsection{Expérimentations animales}

On sait depuis plus de vingt ans, grâce à l'expérimentation animale, que l'irradiation conduit à des cancers mammaires [15]. Les animaux testés ont été les rates Sprague-Dawley et les souris de différentes lignées. Les résultats ont un peu différé selon la prédisposition de l'espèce à faire des cancers spontanés. C'est ainsi que le taux d'induction trouvé voisin de $90 \%$ chez les rates irradiés avec de faibles doses de neutrons est quelque peu faussé par la fréquence spontanée de $50 \%$ de cancers mammaires chez cette souche [19]. Néanmoins, d'autres expériences confirment un effet cancérogène des neutrons beaucoup plus important que celui des gamma et des $X$ et l'efficacité biologique relative des neutrons, pour cet effet, se situerait, selon Vogel, entre 20 et 60 , selon la dose, dans le domaine des faibles doses, et autour de 25 , selon Broerse, selon l'énergie des neutrons $[18,20]$.

Le rôle joué par les hormones dans la cancérogénèse du sein fut démontré dès les années 1932 par Lacassagne. Celui-ci avait notamment montré que la castration inhibait l'apparition de cancer mammaire chez la souris femelle et que, par contre, l'injection de folliculine à des souris mâles induisait des cancers mammaires chez ces dernières, alors que l'hormone mâle exerçait une action inhibitrice [8]. Ces travaux ont ouvert la voie à la cancérologie hormonale ainsi qu'à la thérapeutique de certains cancers (sein, prostate). D'autres auteurs ont confirmé les observations de Lacassagne, et notamment celles de l'effet accélérateur des oestrogènes [13] et de l'effet inhibiteur de la progestérone [6, 14].

Par ailleurs, l'injection de prolactine après une irradiation ou encore la greffe de tumeur hypophysaire conduisent aussi à une exaltation de l'effet cancérogène de l'irradiation [21]. Cette synergie de l'action hormonale s'exerce vis-à-vis de n'importe quel agent cancérogène. Par exemple, l'administration d'un cancérogène chimique tel que le méthylbenzanthracène par voie buccale à des souris jeunes, à l'âge de la puberté, suivie de l'injection d'un oestrogène ou de prolactine, détermine un accroissement spectaculaire de cancers mammaires chez ces animaux, supérieur à celui observé par l'administration du cancérogène seul. Ainsi, l'état physiologique de la glande mammaire au moment où agit le cancérogène est très important, car il peut en amplifier l'effet et la chronologie de l'action du cancérogène se révèle décisive sur l'effet à venir. La prolactine peut jouer des rôles inverses selon qu'elle est administrée avant ou après l'irradiation : d'inhibiteur, elle passe au rôle de potentiateur de l'effet cancérogène [21].

\subsection{Données tirées des enquêtes humaines}

Les enquêtes épidémiologiques apportent sans aucun doute les informations les plus précieuses pour la femme.

Plusieurs enquêtes épidémiologiques menées sur des femmes ayant subi une irradiation ont permis d'estimer l'importance du risque et de déterminer l'influence jouée par certains cofacteurs, capables de moduler la valeur de ce risque. 


\subsubsection{Importance du risque et nature de la relation dose-effet}

Trois enquêtes ont été retenues par les experts parmi l'ensemble des observations qui correspondent à un effectif de femmes suffisant et à un temps de surveillance suffisamment long $[3,10]$. Ce sont :

- l'enquête épidémiologique sur les 60000 femmes survivantes d'Hiroshima et de Nagasaki, prolongée de 1950 à $1974 ; 12000$ d'entre ces femmes avaient reçu plus de 10 rads (de 10 à 450 rads) [17] ;

- l'enquête à New York et Rochester sur les 571 patientes ayant subi une radiothérapie pour mastites aiguës après accouchement (dose moyenne estimée de 247 rads) et suivies pendant 35 ans [10] ;

- l'enquête au Massachusetts sur 1047 femmes tuberculeuses ayant subi des contrôles radioscopiques répétés (nombre moyen : 102 radioscopies) pour pneumothorax, et cumulé ainsi une dose moyenne au sein de l'ordre de 150 rads et suivies 37 ans [4].

D'autres études ont porté sur des effectifs plus restreints ou sur des femmes souffrant d'affections bénignes du sein (fibroadénomes, mastites chroniques ou aiguës, etc.) dont il n'est pas possible d'exclure toute influence sur la cancérisation future ; de ce fait, elles n'ont pas été retenues par les assemblées d'experts, telle celle du BEIR III [3].

Le tableau I, qui reprend l'étude de Land du rapport BEIR III, donne les principales estimations de risque que l'on peut tirer de ces trois enquêtes principales [10].

Les conclusions du BEIR III ont été quelque peu amendées par l'étude critique récente de Land, qui a examiné tous les facteurs tour à tour.

Les trois études concordent sur les points suivants :

1. Le risque est le plus élevé chez les femmes exposées avant l'âge de 20 ans et tend à diminuer ensuite [17]. Une nouvelle analyse récente faite par Land tend pourtant à réduire l'importance de ce facteur, car, à l'exception du Japon, l'excès de l'incidence de cancers a été significatif, encore au-delà de 40 ans [10] ;

2. En moyenne, le risque se situe aux alentours de 9 à 10 cas par million de femme-an et par rad pour celles exposées avant 20 ans et, en moyenne pour tous les âges, entre 6 et 8 cas par million de femmean, par rad. La figure 2 donne les valeurs de ce risque annuel. estimées par les différentes études, en supposant un temps de latence de 10 ans pour les âges d'exposition supérieurs à 20 ans et de l'ordre de 20 ans pour les âges inférieurs ; cela donnerait donc un risque sur la durée de vie d'environ 60 cas par million de femmes exposées à un rad, en moyenne, ce risque étant au maximum de 200 cas par million pour les femmes exposées avant 20 ans. En risque relatif, cela signifie une augmentation par rad du risque spontané de $1 \%$ dans ce dernier cas et de 0,4 à $0,5 \%$ dans la moyenne des cas. II existe donc une assez bonne cohérence des résultats, selon les études, ainsi que le soulignent de nombreux auteurs $[4,10,16]$;

3. Tous ces résultats sont compatibles avec une relation dose-effet linéaire, bien que d'autres relations soient également possibles, dont la linéairequadratique, notamment pour les résultats du Japon, en tenant compte des données dosimétriques révisées pour les deux villes (courbe d, figure 3 ). 
LE CANCER DU SEIN ET L'IRRADIATION : LE POINT DES CONNAISSANCES

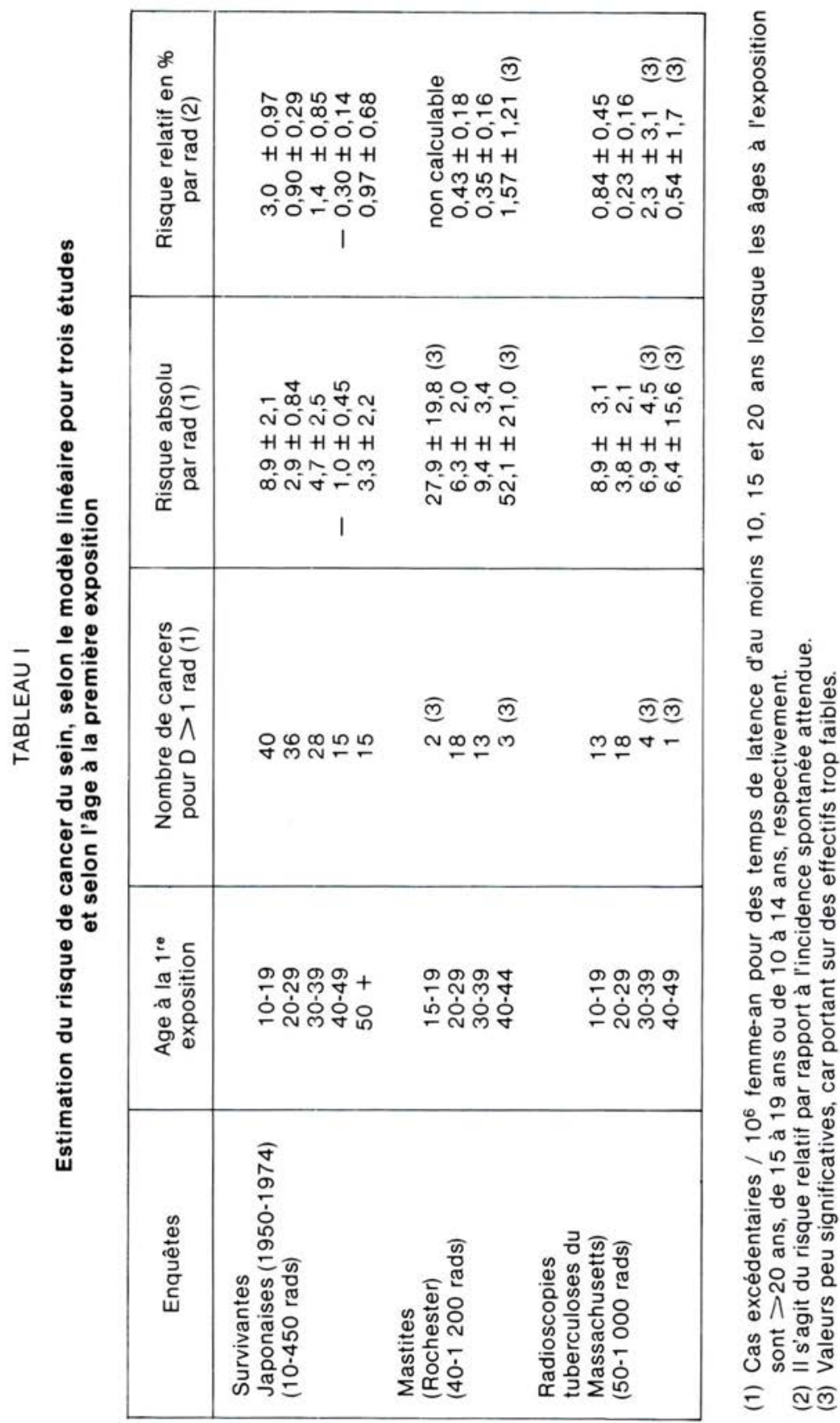




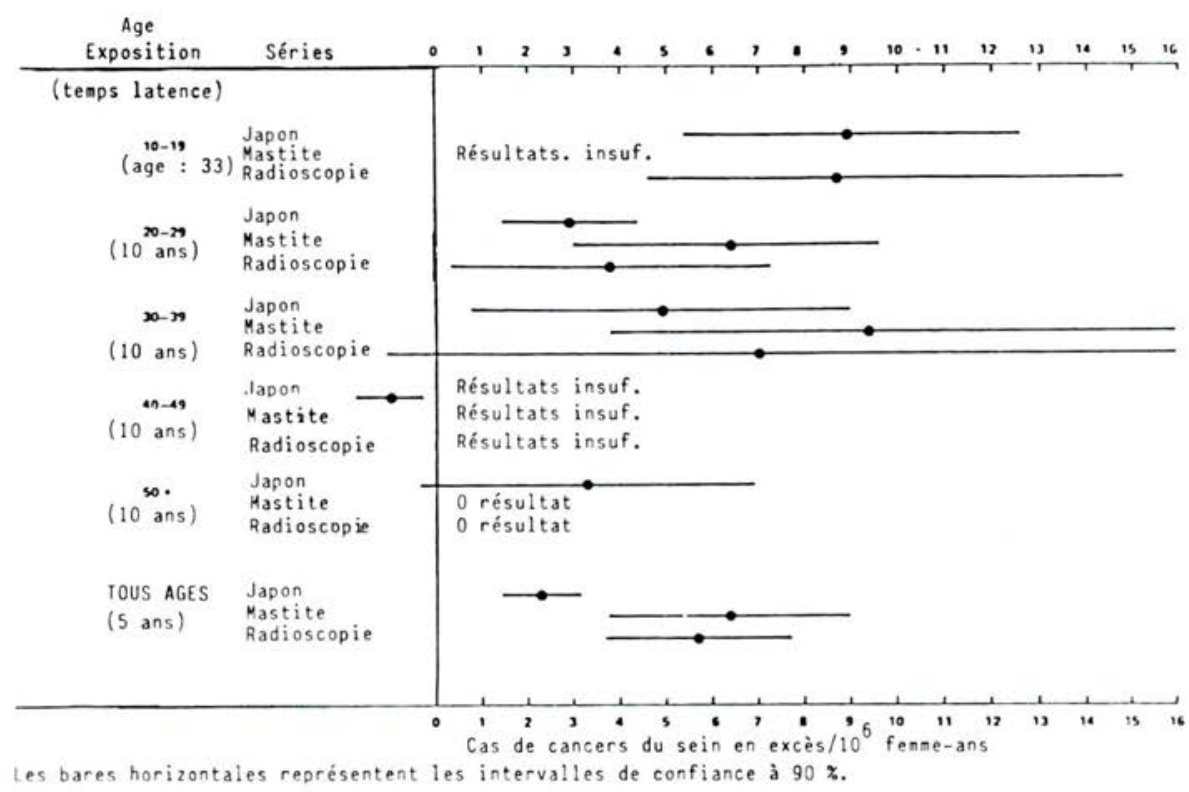

Fig. 2. - Estimations linéaires de risque de cancer du sein par rad après un temps de latence selon l'âge à l'exposition et la série (d'après Land [10]).

A noter que les incidences, aussi bien spontanées que radioinduites, demeurent plus basses chez les japonaises. C'est dans leur groupe aussi que les doses les plus faibles ont été rencontrées et que l'éventail des âges a été le plus large. La figure 3 rassemble les différentes courbes que l'on peut tracer à partir des données de ces enquêtes. Les points sont assortis de leur intervalle de confiance, indiquant le degré d'incertitude du tracé. II semble, néanmoins, qu'une tendance à l'accroissement de l'incidence du cancer du sein apparaisse dès les faibles doses, de l'ordre de 20 rads, tandis qu'au-delà de plusieurs centaines de rads, l'effet létal cellulaire peut entraîner une baisse de l'incidence (courbe b, figure 3).

4. Deux autres faits sont à noter d'après tous ces résultats. D'abord, l'effet ne semble pas influencé par le type de rayonnement, qu'il soit entièrement photonique comme à Nagasaki ou mixte avec des neutrons comme à Hiroshima, puisqu'on n'a pas pu noter de différences pour ce cancer entre les deux villes, aussi bien selon les données dosimétriques anciennes que selon les nouvelles (courbes $c$ et d de la figure 3 ). Cette constatation, en contradiction avec les données de l'expérimentation animale, trouve peut-être son explication dans la très faible proportion de neutrons à Hiroshima.

De même, il semblerait que le débit de dose et le fractionnement de la dose n'exercent pas d'influence sur le taux d'induction, puisqu'une bonne homogénéité des résultats a pu être constatée entre l'irradiation 
aiguë subie par les Japonaises et l'irradiation fractionnée sur plusieurs mois subie par les tuberculeuses par radioscopies $[5,16]$. Le sein serait donc moins apte qu'un autre tissu à réparer et par conséquent les effets de petites doses répétées seraient cumulables à son niveau.

Ce qui semble appuyer ce point de vue est la tendance constatée à l'accroissement de l'incidence de cancer du sein chez les femmes peintres en cadrans lumineux, américaines et britanniques, exposées pendant plusieurs décennies au risque du radium [1, 2]. La dosimétrie externe de ces femmes manque généralement. Chez les britanniques qui ont été contrôlées, le débit de dose moyen, entre 1939 et 1950, était de 4 mGy par semaine $(0,4$ rad). Mais il est difficile d'attribuer l'excès de cancers constaté à l'irradiation externe plus qu'à l'irradiation interne due au radium. L'étude doit se poursuivre afin de confirmer ces résultats.
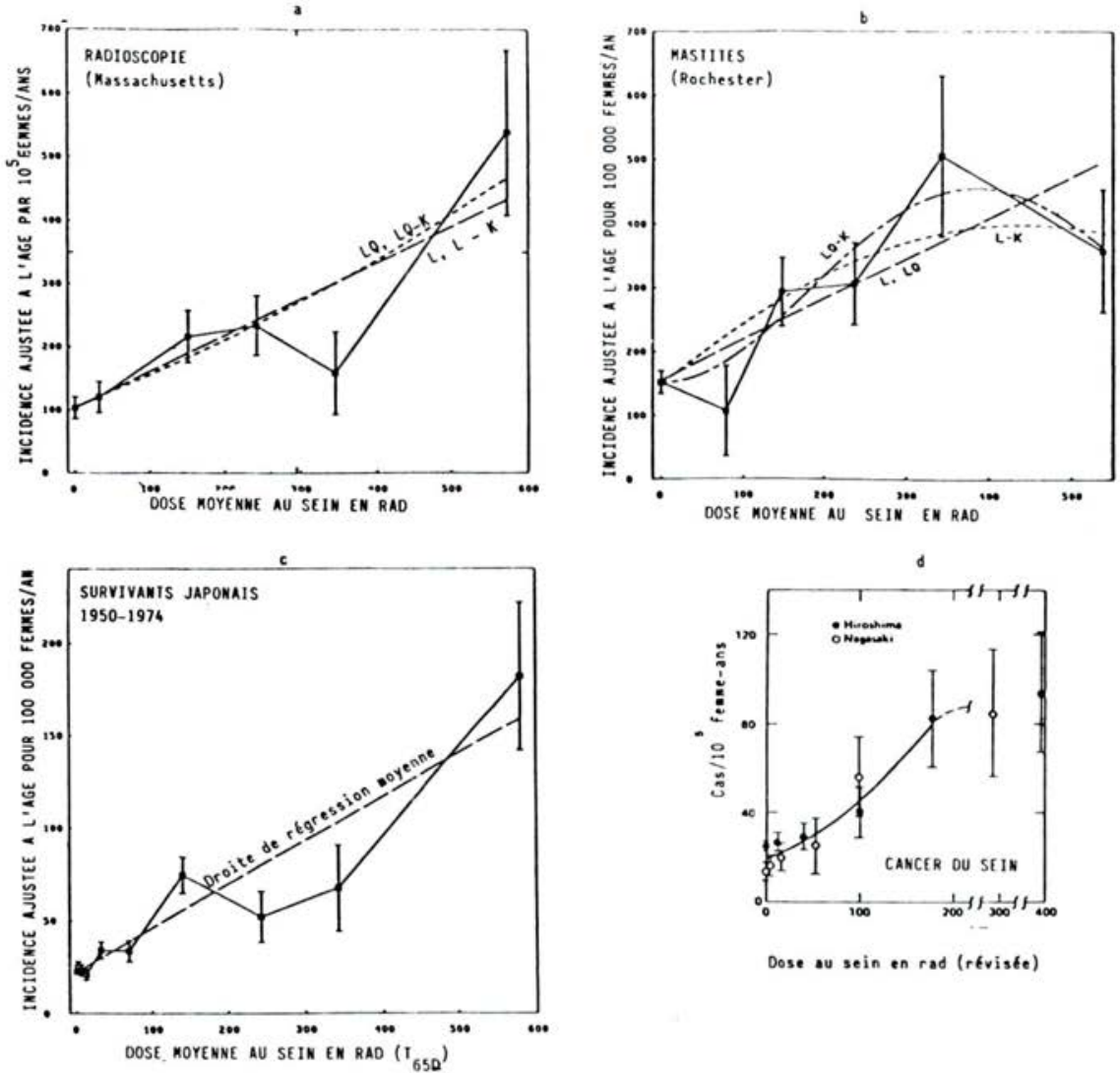

Dose su sein en rad (rtuiste)

Fig. 3. - Différentes relations entre l'incidence de cancers et la dose au sein (d'après Straume et Dobson, Health Phys. 1981, 41, p. 666).

Les barres d'erreurs sont les limites à $50 \%$
L : linéaire
L-K : linéaire tenant compte de l'effet létal.
Lq : linéaire-quadratique.
Lq-K: linéaire - quadratique tenant compte de l'effet létal. 
5. Enfin, les cancers du sein radioinduits n'apparaissent pas plus tôt que les cancers spontanés. Le temps de latence observé est toujours supérieur à 5 ans et peut dépasser 30 ans (fig. 4), le temps moyen étant d'environ 20 ans. On relève une augmentation, aux mêmes âges, de l'incidence des cancers spontanés et des cancers radioinduits $[3,10$, $16,17]$.

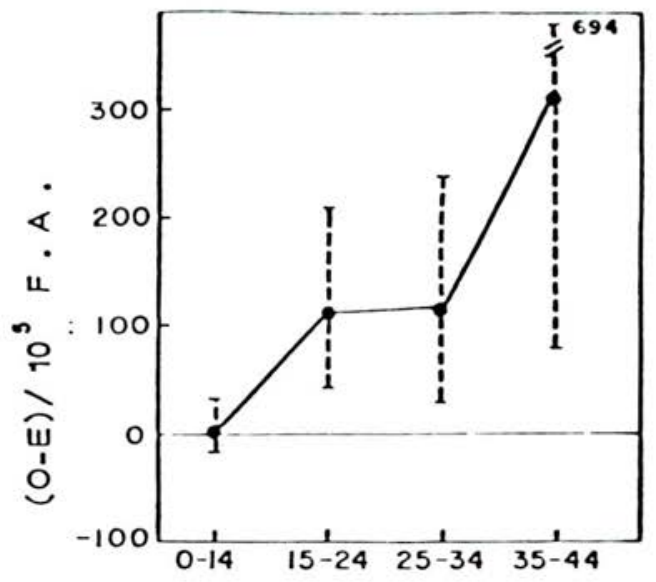

Temps depuis la 1ère exposition

Fig. 4. - Cancers du sein excédentaires selon le temps

depuis la première exposition (toutes doses confondues) (d'après J. Boice, In : Carcinogenesis, Oxford, Pergamon Press, 1979).

\section{CO-FACTEURS CANCEROGENES PARTICULIERS A LA GLANDE MAMMAIRE}

La radiosensibilité de la glande mammaire et sa susceptibilité au cancer sont en relation avec les caractéristiques propres à ce tissu. Or, ses caractéristiques physiologiques sont éminemment évolutives au cours de la vie de la femme, et plus précisément au cours de sa vie génitale. Le tissu, quiescent durant la première décennie de la vie, entre en croissance juste avant la puberté sous l'effet de l'hormone oestrogénique, la folliculine, secrétée par suite d'une stimulation hypophysaire. II existe pour cela dans la glande mammaire des récepteurs hormonaux sélectifs, sortes de relais de l'action in situ [12].

La plupart des enquêtes épidémiologiques montrent que le facteur déterminant est l'âge de l'exposition : la plupart des groupes exposés avant l'âge de 20 ans ont manifesté le risque le plus élevé (fig. 5). Cette observation coîncide avec celle effectuée lors des expérimentations animales [13]. Les périodes pubertaire et postpubertaire très proches demeurent les plus radiosensibles pour le sein. Elles coïncident avec le moment où le tissu prolifère et où l'imprégnation oestrogénique de la glande est maximale. II est possible que durant cette période, où les cellules sont peu différen- 
ciées et en voie de croissance, la faculté de réparation de l'ADN lésé sous l'effet d'un cancérogène soit amenuisée, et que des cellules lésées subsistent, rendues vulnérables à l'action ultérieure d'un promoteur du cancer.
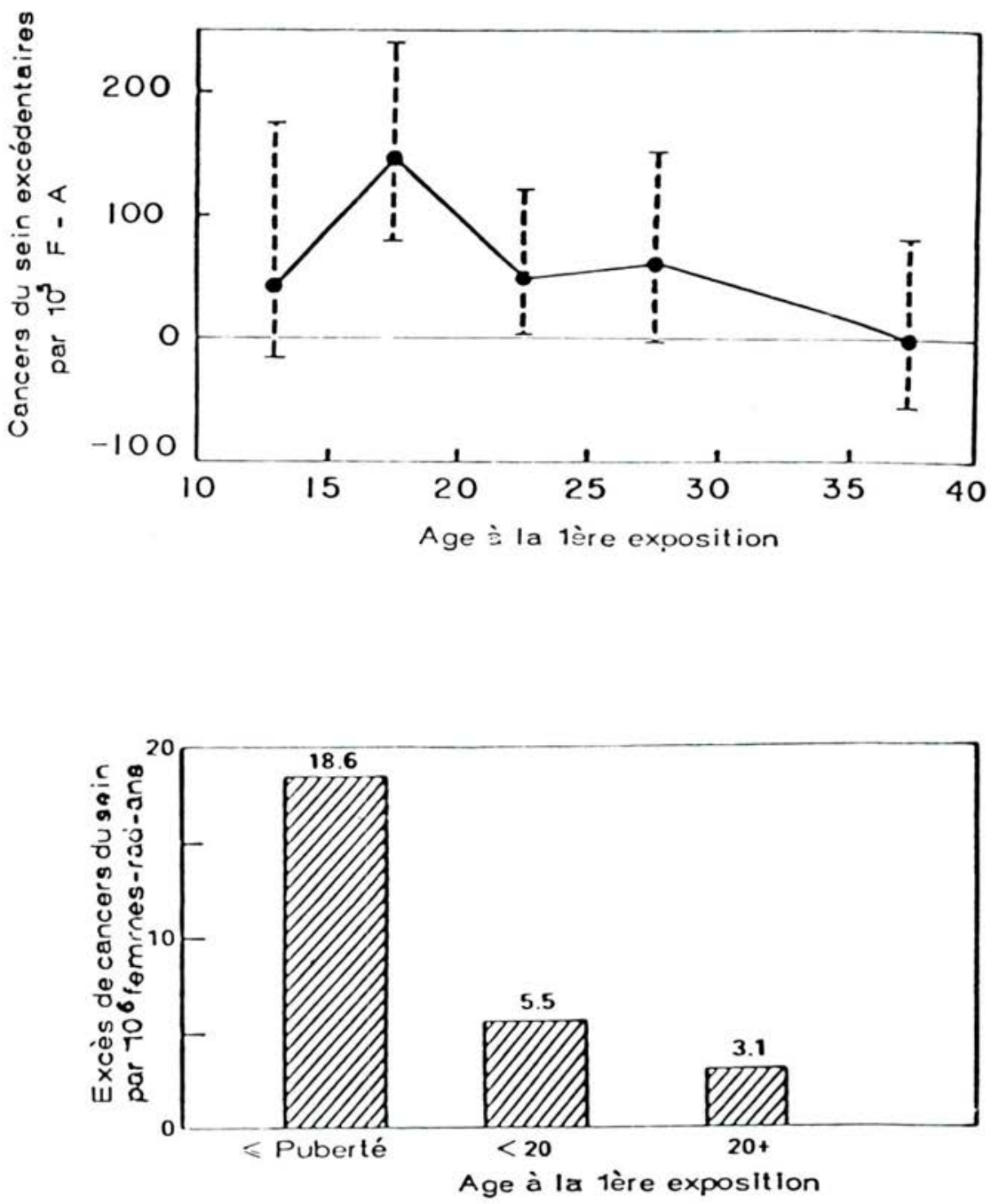

Fig. 5. - Cancers du sein excédentaires en fonction de l'âge à la première exposition (d'après J. Boice, In : Carcinogenesis, Oxford, Pergamon Press, 1979). II s'agit des données, toutes doses confondues et pour toute la durée d'observation. 
De nombreux facteurs peuvent donc interférer et la question de la cancérogénèse du sein s'avère fort complexe.

Une autre période apparaît très sensible : celle de la grossesse, où l'imprégnation hormonale est très élevée, notamment en fin de grossesse, où intervient de plus la sécrétion de prolactine : c'est aussi une période de profondes modifications physiologiques de la glande [12].

En réalité, toutes les étapes de la vie sexuelle de la femme interviennent pour inhiber ou favoriser, selon les cas, la cancérogénèse du sein. L'âge de la puberté, l'âge à la première maternité, le nombre de naissances et d'allaitements, l'âge à la ménopause sont autant d'éléments qui interfèrent sur la susceptibilité de la glande à se cancériser. De plus, la prédisposition héréditaire, le niveau socio-économique, l'alimentation, l'exposition à diverses nuisances physiques ou chimiques peuvent intervenir.

L'âge à la première naissance, notamment, a fait l'objet d'une étude, mettant en évidence la corrélation entre l'âge tardif de la première naissance et le degré de risque (fig. 6) [4, 11].

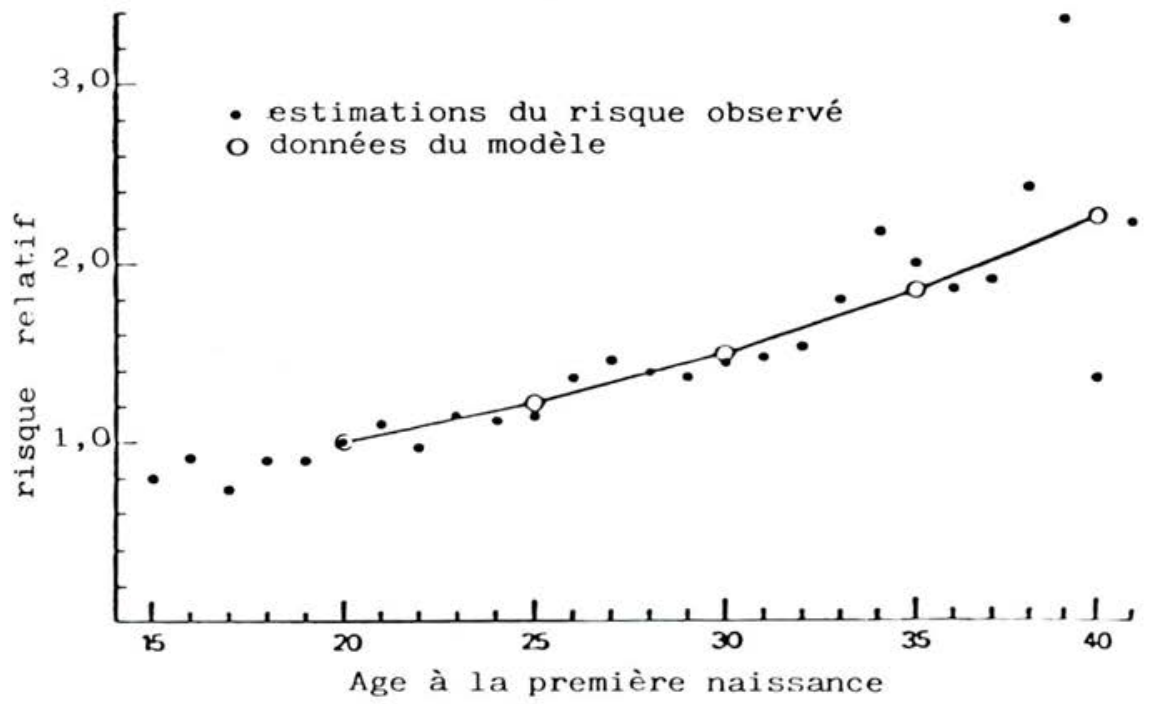

Fig. 6. - Risques relatifs de cancer du sein, selon l'âge de la première grossesse, rapportés au risque de la première grossesse à 20 ans [17].

Les femmes sans enfant semblent plus sensibles à la radio-cancérogénèse du sein que celles ayant enfanté, ainsi que cela a été observé notamment sur les femmes peintres en cadrans lumineux [1]. Mais la réceptivité de la glande mammaire aux hormones diffère d'une femme à l'autre, si bien que le rôle joué par celles-ci dans l'induction du cancer du sein n'est pas toujours aussi net. II apparaît globalement une relation étroite entre la fréquence de cancers du sein dans une population et le degré de son niveau socio-économique. En effet, on observe que, plus ce niveau est élevé, plus l'alimentation est riche et abondante, et aussi plus 
la fécondité baisse et ces facteurs semblent favoriser l'incidence du cancer du sein, alors que c'est l'inverse parmi les populations à faible niveau socioéconomique.

\section{DISCUSSION}

Comme dans tout processus de cancérogénèse, beaucoup d'inconnues subsistent quant au mécanisme de la radioinduction du cancer du sein. Moolgavkar et coll ont proposé un modèle mathématique à deux étapes [11], qui est intéressant, mais qui, comme tous les modèles, a sans doute le défaut d'être trop rigide et de ne pas être applicable à tous les cas. Ce modèle a été repris et critiqué récemment par d'autres [7]. Selon ces derniers, bien que la relation dose-effet la plus plausible pour les populations irradiées soit la linéaire, ce qui est en faveur d'un processus à un seul événement, le long temps de latence du cancer du sein (plus de 20 ans) plaide aussi en faveur de l'intervention d'autres facteurs intermédiaires [9].

Ainsi, l'irradiation serait un initiateur, avec une réponse à un seul coup, qui exigerait ensuite une altération additionnelle pour induire le cancer. Cette promotion pourrait être le fait ou d'un agent "endogène" hormonal, ou "exogène" soit chimique (en provenance de l'alimentation, par exemple)*, voire viral (cela n'a pas reçu jusqu'à présent de vérification, contrairement à ce qui existe chez les murins). L'existence de tumeurs du sein de natures histologiques différentes et de sensibilités très différentes aux hormones permet de penser que leurs étiologies sont très différentes. La proportion des tumeurs demeurées sensibles aux hormones apparaît identique parmi les femmes non ménopausées et les femmes ménopausées. Cela semble indiquer une même cinétique d'induction pour ces deux séries de cancers et si une différence apparaît, ce serait au niveau de la nature même des événements initiateurs. II est possible aussi qu'il existe des variantes selon la nature des agents impliqués et les probabilités d'événements promoteurs, par exemple le taux de prolifération des cellules transformées. L'action favorisante des oestrogènes pourrait alors se limiter à la stimulation qu'ils exerceraient sur cette multiplication cellulaire.

\section{CONCLUSION}

Le sein se trouve au carrefour des effets de tous les cancérogènes connus. Notamment, il semble qu'il soit sensible aux cancérogènes chimiques, dans la mesure où il renferme des cellules à haute activité enzymatique, capables de métaboliser des procancérogènes en cancérogènes pouvant ensuite agir localement. L'irradiation s'est révelée sans conteste cancérogène pour le sein, ainsi que le montrent de nombreuses enquêtes épidémiologiques.

Les probabilités d'action d'un agent cancérogène sur le sein sont accrues par la présence de facteurs endogènes facilitants. Notamment, les oestro-

* Les graisses ayant été accusées de pouvoir exercer un rôle favorisant, par oxydation des acides gras. 
gènes semblent jouer un rôle promoteur important, l'ensemble des enquêtes épidémiologiques tendant à montrer que l'âge où le risque est le plus élevé coïncide avec la période pubertaire ou proche de cette période. Bien évidemment, de nombreux autres facteurs personnels entrent en jeu pour pondérer cette action hormonale.

Les implications de ces enseignements sont essentiellement médicales, notamment à propos de l'opportunité d'effectuer des examens mammographiques systématiques chez les femmes jeunes [16]. Quant aux expositions professionnelles, elles sont réglementées par les limites de doses recommandées par la CIPR. Celle-ci a affiné dernièrement sa doctrine en tenant compte des dernières données, et en estimant à 100 cas par million de femmes le nombre total de cancers du sein guérissables ou non induit par une exposition à $1 \mathrm{rem}$. Ce risque est donc important, car il constitue la moitié du risque total radioinduit. Notamment, il semble souhaitable d'exclure de l'exposition les femmes jeunes, enceintes ou en train d'allaiter.

\section{BIBLIOGRAPHIE}

[1] ADAMS E., BRUES A. Breast cancer in female radiumdial workers first employed before 1930. J. Occ. Med., 1980, 22, 583-587.

[2] BAVERSTOCK K.F., PAPWORTH D., VENNART J. Risks of radiation at low dose rates. Lancet, $1981,1,430-433$.

[3] BEIR III. Committee on the biological effects of ionizing radiations. Washington : National Academy of Sciences, National Research Council, 1980.

[4] BOICE J.D., ROSENSTEIN M., TROUT E.D. Estimation of breast doses and breast cancer risk associated with repeated fluoroscopic chest examinations of women with tuberculosis. Radiat. Res., 1978, 73, 373-390.

[5] BOICE J.D., LAND C.E., SHORE R.E., NORMAN J.E., TOKUNAGA M. Risk of breast cancer following low-dose radiation exposure. Radiology, 1979, 131, 589-597.

[6] CRONKITE E.P., SHELLABARGER C.J., BOND V.P. et al. Studies on radiationinduced mammary gland neoplasia in the rat. I. The role of the ovary in, the neoplastic response of the breast tissue to total - or partial - body $\mathrm{x}$-irradiation. Radiat. Res., 1960, 12, 81-93.

[7] EMMELOT P., SCHERER E. Two-stage model for breast carcinogenesis in females. J. Nat. Canc. Inst., 1981, 67, 72-74.

[8] LACASSAGNE A. Apparition de cancers de la mamelle chez les souris mâles après une injection de folliculine, C.R. Acad. Sci., 1932, 195, 630.

[9] LAND C.E., MC GREGOR D.H. Temporal distribution of risk after exposure. In : Prevention and detection of cancer Part I - Prevention, Vol. I - Etiology (Nieburgs H.E., Ed.). New York: M. Dekker, 1977, 831-843.

[10] LAND C.E., BOICE J.D., SHORE R.E., NORMAN J.E., TOKUNAGA M. Breast cancer risk from low-dose exposure to ionizing radiation : results of parallel analysis of three exposed populations of women. J. Nat. Cancer Inst., 1980, 65, 353-376.

[11] MOOLGAVKAR S.H., DAY N.E., STEVENS R.G. Two-stage model for carcinogenesis : epidemiology of breast cancer in females. J. Nat. Cancer Inst., 1980, 65, 559-570.

[12] SAEZ S. Hormones stéroîdes dans le cancer du sein chez la femme. Conférence à la $7{ }^{\circ}$ réunion d'étude, Cancer-environnement et géocancérologie, Paris, 8 octobre 1981.

[13] SEGALOFF A., MAXFIELD W.S. The synergism between radiation and estrogen in the production of mammary cancer in the rat. Cancer Res., 1971, 31, 166-168. 
[14] SEGALOFF A. Inhibition by progesterone of radiation-estrogen-induced mammary cancer in the rat. Cancer Res., 1973, 33, 1136-1137.

[15] SHELLABARGER C.J., CRONKITE E.P., BOND V.P. et al. The occurence of mammary tumors in the rat after sublethal whole-body irradiation. Radiat. Res., 1957, 6, 501-512.

[16] SHORE R.E. Application of epidemiologic studies of radiation induced breast cancer to mammographic screening guidelines. In Radiation research, 6 . intern. congress, Tokyo, May 13-19, 1979 (OKADA S. et al., Eds). Tokyo, JARR, 1979,973-979.

[17] TOKUNAGA M., NORMAN J.E., ASANO M., TOKUDA S., EZAKI H., NISHIOMORI I., TSUJI Y. Malignant breast tumors among atomic bomb survivors Hiroshima and Nagasaki, J. Nat. Canc. Inst. 1979, 62, 1347-1359.

[18] VAN BEKKUM D.W., BROERSE J.J. et al. Radiation induced mammary cancer in the rat. In: Radiation research, 6 . intern. congress, Tokyo, May 13-19, 1979 (OKADA S. et al., Eds). Tokyo, JARR, 1979, 743-752.

[19] VOGEL H.H. Jr. Mammary gland neoplasms after fission neutron irradiation. Nature, $1969,222,1279-1281$.

[20] VOGEL H.H., ZALDIVAR R. Neutron-induced mammary neoplasms in the rat. Cancer Res., 1972, 32, 933-938.

[21] YOKORO K., SUMI C., ITTO A., HAMADA K. KANDA K., KOBAYASHI T. Mammary carcinogenic effect of low-dose fission radiation in wistar / furth rats and its dependency on prolactin. J. Nat. Cancer Inst., 1980, 64, 1459-1466. 